\title{
CONTACT IDENTIFICATION FOR ASSEMBLY/DISASSEMBLY PROCESS IN CAD-CAE SOFTWARE
}

\author{
POPA, C[icerone] L[aurentiu]; PARPALA, R[adu] C[onstantin] \& COTET, C[ostel] E[mil]
}

\begin{abstract}
Assembly/Disassembly (A/D) simulations are important for improving designs and efficiency of product development processes. Although all CAD software have some Assembly/Disassembly simulation capabilities a specialized CAD platform is not available. This paper contains a comparative study between main CAD-CAE software from the assembly / disassembly perspective. The results of the study will be used in order to propose a simulation platform that will contain the main function and facilities needed for Assembly/Disassembly studies and analysis.
\end{abstract}

Keywords: assembly / disassembly / CAD /CAE / simulation

\section{INTRODUCTION}

The ability to perform A/D analysis of a multicomponent assembly is helpful for the design of new products and for the reuse/recycling of the assembly. In order to perform such a complex analysis a suite of software tools is necessary. Sometimes these tools are not available in all softwares. The main purpose of this paper is to analyse all the utility tools available in current CAD programs in order to include them in an A/D CAD design platform.

\section{SOLID WORKS}

Solid Works is a 3D design software developed by Dassault Systèmes. The complete design platform contains: 3D design software, analysis software and product data management software. This tool makes it possible for mechanical designers to quickly sketch ideas, experiment with features and dimensions, and produce models and detailed drawings.

Commercial Computer Aided Design (CAD) software like Solid Works offer the possibility to conceive part models and assembly models. This software considers an assembly model as the result of correct spatial arrangements of the components. In order to easily create such arrangements, the designer can specify a set of standard constraints like: coincident, parallel, perpendicular, tangent, concentric, lock, distance or angle. These include contact mates between flat and/or cylindrical surfaces, alignment and orientation constraints. Different combinations of constraints can produce the same result (position of the components) and these constraints do not express directly the relative motion possible between components.

Besides the classical constraints, Solid Works software also includes two additional set of advanced mating features: advanced mates and complex mechanical mates. The advanced mates are deployed in order to model the contacts and to partially reproduce the real movements allowed.

For example, linear coupler mate can represent a linear guide, hinge mate can describe a pin contact and a screw mate can describe a helical contact. The complex mates can also be used for constraining some complex surfaces. Solid Works contains some assembly analysis functions: interference detection and clearance verification. One related function is clearance verification used to check the clearance between different components in assemblies. The software checks the minimum distance between the components and reports clearances that fail to meet the minimum acceptable clearance specified [6].

Solid Works platform contains, beside the standard CAD program, two analysis modules: Motion and Simulation. These embedded software offer conversion functions of geometric constraints in kinematic linkages, thereby reducing duplication, but only for a part of constraints - some complex mates must be manually redefined before the analysis.

When assembly models are imported from another CAD system in Solid Works, the mates must be redefined in order to have a complete representation of the product model. Similarly, when a Solid Works assembly model is exported to a kinematic analysis system, e.g. Adams, the kinematic links between the components must be specified.

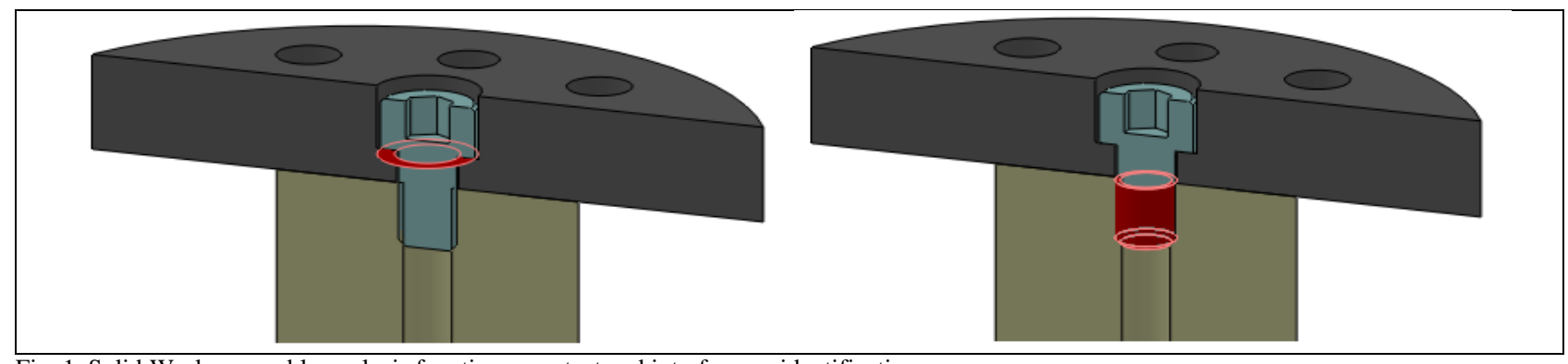

Fig. 1. Solid Works assembly analysis function - contact and interference identification 
The information about geometrical mates is not used to define the relative mobility of the components and the proposed constraints are related to the position of the components but do not explicitly represent the contacts between components. Therefore, this type of information is interesting but its effectiveness is limited and its transfer through standards data exchange is not currently possible.

The positional constraints refer only to the reciprocal position of components and these data are neither sufficient nor consistent with the requirements for the identification of contacts, these data are not intrinsic to the definition and characterization of the contacts and their commune zone associated.

Following this analysis, one can conclude that Solid Works is a 3D modeling program that offers many design features but its main focus is on part design, thus missing assembly analysis functions.

\section{CATIA V5 R19}

CATIA V5 R19 is an integrated CAD-CAM-CAEPLM CATIA V5 R19 system produced by Dassault Systems. While in Assembly Design the user can perform only clash, contact and interference analyses on objects in static positions, Digital Mock-up applications (DMU Fitting and DMU Kinematics) covers the analyses such as clash between objects as they are assembled/disassembled or clash analysis between assembly parts as they operate [3].

Assembly Design workbench allows the user to create assembly models inserting components (i.e. parts or sub-assemblies) in a product by setting different constraints between them, but also to design new parts in the assembly context.

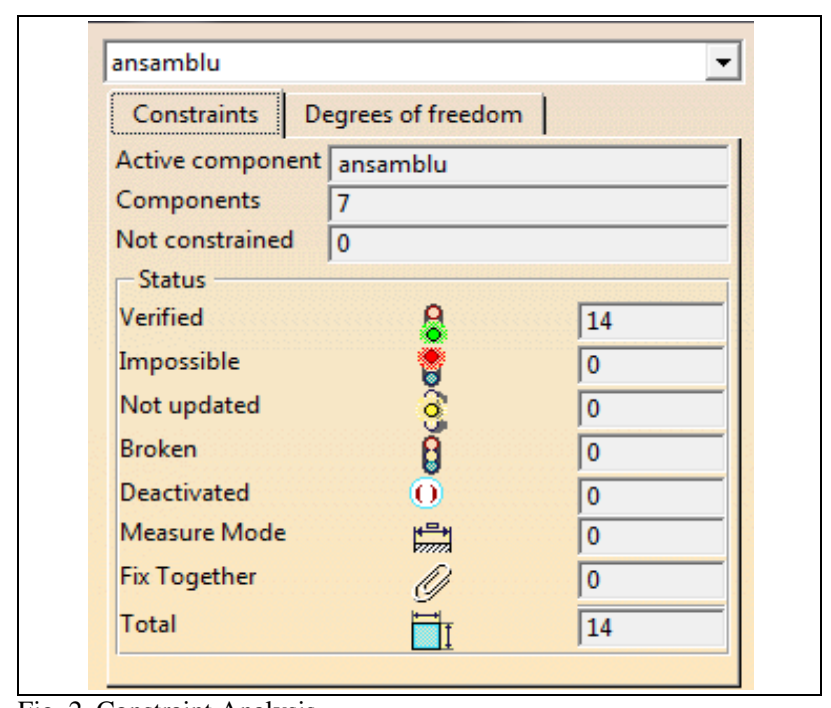

Fig. 2. Constraint Analysis

In the same manner as in other CAD software, in CV5 Assembly Design the user places the components by establishing their reciprocal spatial position using standard constraints, one of the components being declared fixed.

Quick Constraints option places the first possible constraint between selected geometry: point, plane, line or circle. This first possible constraint is established from a priority list which can be set by the user.
For some types of selected geometry, Contact, Offset and Coincidence constrains are redundant, as they produce the same result. In order to avoid placing constraints between wrong elements or for making the elements selection easier for user, CV5 offers the possibility to select only published geometry.

Regarding the analyses available in Assembly Design workbench, along clash/contact/ interference analysis, constraint and DOF analyses can be performed. The interference analysis visually informs the user on the interference area and displays the interference vector and matrix of conflicts, which can be exported in xml format. Deeper investigations are necessary in this regard [1].

However, constraint analysis does not indicate overconstrained or inconsistent assemblies. This information can be obtained using Dependencies option, also available in the Analyze toolbar. Degree of freedom option analyses and displays the translation and rotation DOFs of the active component [2].

Resuming, the tools available in the Assembly Design workbench allow to spatial position assembly components relative to each other. The analyses are performed considering the components as static.

DMU Fitting is not an assembly constrained-based application, but the components have to be placed in their correct functional position at the beginning of the simulation usually using constraints. The movements are made according to a track/tracks specified by the user, generating the disassembly trajectory. Automation of the disassembly can be performed through Visual Basic macro programming. The movement trajectories are defined frame by frame in an interactive manner, avoiding clashes and respecting movements' limitations.

DMU Kinematics has an option - Assembly Constraints Conversion, which allows inheriting constraints from the assembly. For this option, the software performs an analysis of each of the assembly constraints and suggests a kinematic equivalent constraint. The conversion of constraints in joints is based on an analysis of the type o geometry involved. For instance, selection of a pair of components and: Line/Line assembly constraint produces a Coincidence joint, Line/Line and Plane/Plane produce a Revolute joint; selection of three components produces a Rigid kinematic joint, etc.

Similar to other 3D CAD software, CATIA V5 assembly application uses geometrical elements such as points, lines, planes or surfaces for defining the relative position of two parts. The functional role of the components (or their surfaces) is not considered in the assembly modelling process, nor the contact zones between components.

The A/D operations can be simulated only in DMU Fitting by generating movements' tracks. However, these tracks are defined by the user, (who knows exactly how the assembly should work and which are the reciprocal functional constraints between parts), after their initial placement of the assembly components in their functional positions, the software not offering any information, assistance nor suggestions regarding possible solutions for A/D operation. 


\section{SOLID EDGE ST2}

Solid Edge with Synchronous Technology is a computer-aided design (CAD) system for mechanical assembly, part modelling, and drawing production developed by Siemens. Using STREAM technology, Solid Edge is designed to increase software performance with an interface that ensures maximized user productivity.

The Assembly environment allows users to create assembly relationships between parts. These relationships are automatically maintained throughout the development of the design in order to preserve the design intended. Solid Edge accommodates the fact that most parts are designed in the context of an assembly. To support this workflow, Solid Edge provides tight integration with the part modelling environments, visualization tools, data management tools, and part-topart relationship management tools.

One can build an assembly using existing parts and subassemblies, or model new parts within the context of the assembly. The user can even use parts that were not modelled with Solid Edge.

Solid Edge provides several workflows for positioning parts in an assembly:

- Flash Fit

- Traditional Workflow

- Reduced Steps

- Capture Fit

In Solid Edge assemblies the user can create exploded views, renderings, and animations using the ExplodeRender-Animate (ERA) application [5].

With ERA the following actions are possible: exploding assemblies automatically; exploding parts within an assembly manually; repositioning parts within an exploded view; moving parts within an exploded view and creating an animation of the Assembly/Disassembly (A/D) process. All this options are based on the relationships applied between parts.

\section{ANSYS}

ANSYS is a general purpose finite element modelling package for numerically solving a wide variety of mechanical problems. These problems include: static/dynamic structural analysis (both linear and nonlinear), heat transfer and fluid problems, as well as acoustic and electromagnetic problems.

ANSYS connections are used to model relations between assembly parts. ANSYS connections include: contact regions, joints, springs and weld points.

Contact conditions are formed where bodies meet. Contact types are characterized by their translational and tangential stiffness. There are no limits placed on the number of bodies that comprise an assembly and depending on the type of contact, the analysis can be linear or nonlinear. A nonlinear analysis can increase runtime significantly, as the solver will internally run iterations to arrive at a converged solution.

As a main difference from CAD software the initial contact information can be analyzed in order to correctly define global contact settings. This information contain: contact side (Contact or target), contact type, status (far open, open, closed), penetration, gap, geometric gap, geometric penetration, Normal stiffness, tangential stiffness etc.

There are also some results regarding contact behaviour that can be obtained. Those results are: Frictional stress, gap, penetration, pressure, sliding distance, status. Scoping method affect the way that results are displayed. If the contact tool is scoped using geometry selection method the result will be averaged on the scoped geometry else the result will be displayed on target contact pair. The difference in contact settings determine how the contact bodies can move relative to one other. Most of these contact types only applies for surface to surface contact.

The contact type depends on the type of the problem. If contact can separate or open slightly a nonlinear contact (frictional, frictionless or rough) must be used. The contact type depends on the type of the problem. If contact can separate or open slightly a nonlinear contact (frictional, frictionless or rough) must be used. However using those contact types can result in a longer solver times and can cause converging problems. Usually nonlinear contacts must be solved with well-defined meshes.

In ANSYS a joint is classified as a remote boundary condition. A joint can be scope to single or multiple faces. The scoping can either be from body to body or body to ground. For body to body scoping there is a reference and a mobile side. For body to ground scoping the reference side is assumed to be grounded; scoping is only available on mobile side. For body-to-body joint scoping can be reversed between the Reference and Mobile sides.The scoping of a joint must be accompanied by the definition of a joint coordinate system. This coordinate system defines the location of the joint. It is imperative that the joint coordinate system to be fully associative with the geometry, otherwise, the coordinate system could move in unexpected way [4].

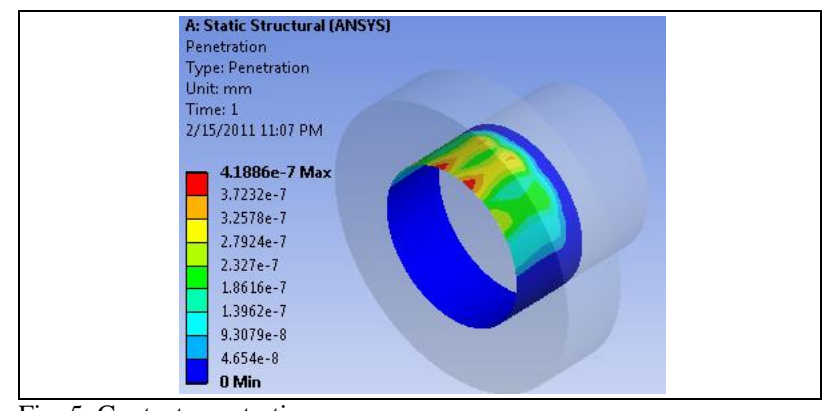

Fig. 5. Contact penetration

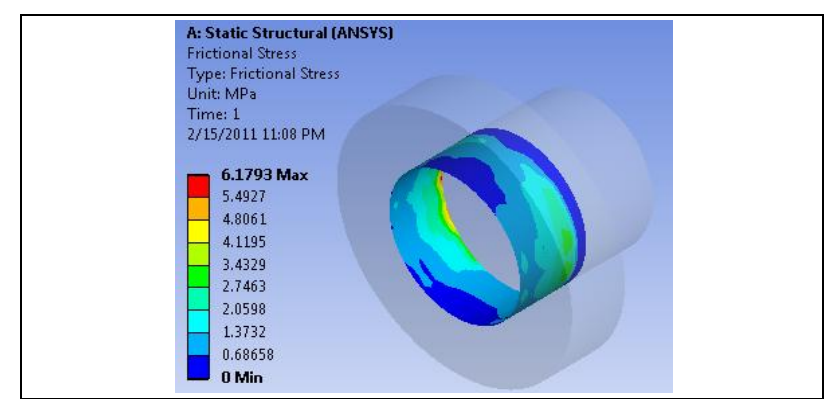

Fig. 6. Contact frictional stress 
Unlike in others simulation systems Stops and Locks can be defined. Stops and Locks are optional constraints that may be applied to restrict the motion of the free relative degree(s) of freedom (DOF) of most types of joints. Any analysis that includes a valid joint type can involve Stops and/or Locks. For the applicable joint types, you can define a minimum and maximum (min, max) range inside of which the degrees of freedom must remain.

A Stop is a computationally efficient abstraction of a real contact, which simplifies geometry calculations. For Stops, a shock occurs when a joint reaches the limit of the relative motion. A Lock is the same as a Stop except that when the Lock reaches the specified limit for a degree of freedom the Lock becomes fixed in place.

Once joints are created, fully defined, and applied to the model, a Joint DOF Checker calculates the total number of free degrees of freedom. The number of free degrees of freedom should be greater than zero in order to produce an expected result. If this number is less than 1 , a warning message is displayed stating that the model may possibly be over constrained, along with a suggestion to check the model closely and remove any redundant joint constraints.

Over constrained conditions can occur when more constraints than are necessary are applied to a joint's degrees of freedom. These conditions may arise when rigid bodies are joined together using multiple joints. The over constraints could be due to redundant joints performing the same function, or contradictory motion resulting from improper use of joints connecting different bodies.

The Contact Tool allows one to examine contact conditions on an assembly both before loading, and as part of the final solution to verify the transfer of loads (forces and moments) across the various contact regions.

Incorrect contacts can be corrected by using some advanced settings like adjust to touch or add offset. The Assemble tool performs the assembly of the model, finding the closest part configuration that satisfies all the joints.

Unlike contacts joints are not so well implemented in ANSYS. There are only a few analyses types that use joints (Transient structural). Joints are characteristic to Multi-body Dynamics (MBD) analyses and are used to model the kinematic behavior of bodies.

\section{CONCLUSION}

Generally, mechanical products are designed using a standard industrial CAD modeller conform to the characteristics mentioned above. According to analyses made in this work, the Solid Works platform is interesting and useful, but it lacks strong properties for the description and analysis of assemblies. Another conclusion is that these environments express the mobility between components only for an operational configuration of a product, but an Assembly/Disassembly (A/D) process requires the mobility model between all components and all trajectories between components because all of them are related potential solutions for an A/D operation.
Almost all CAD modellers include functions for relative positioning of components and contain assembly modules which offer the possibility to define constraints between components. These constraints are limited to the specification of the relative position of surfaces, or axes related to each component, but not necessarily attached to the contact surfaces between components.

Unlike CAD software, FEM software defines contact regions as functional geometry. As an example in ANSYS connections types take into consideration body boundaries. Using some advanced techniques contact regions can be defined and exported into CAD software for further analysis.

The proposed simulation platform will provide an intelligent tool to aid engineers in the design process. In order to decrease the design time and the test costs, this platform will offer the possibility to automatically identify contacts from a mechanical system, to compute the mobility of a component form an assembly, to determine the best sequence of mounting/dismounting of a product.

This simulation environment will be able to collaborate with standard CAD software and with real time simulation platforms. The main beneficiaries of the results of this project are companies from industry. Thus, the simulation platform could be used in any mechanical design department.

\section{ACKNOWLEDGEMENTS}

This work was supported by CNCSIS-UEFISCSU, project number PN-II RU 233/2010, project title: "Assembly/Disassembly Process Modelling", project type: "Research projects for stimulation of the founding/forming of young independent research teams".

\section{REFERENCES}

[1] Jayaram, S., Jayaram, U., Kim, Y.J., de Chenne, C., Lyons, K.W., Palmer, C., Mitsui, T., (2009), Industry case studies in the use of immersive virtual assembly, Virtual Reality Journal, vol. 11, pp.217-228, Ed. Springer, 2009

[2] Peng, Q., Lee, H., (2005), A case study of product design for assembly using an integrated method, Conference ASME-DETC, Long Beach - USA

[3] Popescu, D., Popa, C.L., Ciobanu, L.F., Grigoroiu, G.E., Constantinescu, C.C., (2004), Indrumar CAD CATIA V5R8, ISBN 973-700-011-0, Editura AIUS, Craiova, Romania

[4] Pupaza, C., Parpala, R.C., (2011), Modelare și analiză structurala cu ANSYS Workbench, Editura Politehnica Press, București, Romania

[5] Solid Edge ST2 User Manual

[6] Solid Works 2009 User Manual 